Revue de recherche interdisciplinaire sur le genre et la sexualité

$37 \mid 2020$

Le rideau déchiré

\title{
La sex research : émergence, développement et éclatement d'une communauté épistémique
}

Alain Giami

(2) OpenEdition

Journals

Édition électronique

URL : https://journals.openedition.org/sextant/338

DOI : $10.4000 /$ sextant.338

ISSN : 2795-8736

Éditeur

Éditions de l'Université de Bruxelles

\section{Édition imprimée}

Date de publication : 1 décembre 2020

Pagination : 149-172

ISBN : 978-2-8004-1744-8

ISSN : $1370-267 X$

Référence électronique

Alain Giami, «La sex research : émergence, développement et éclatement d'une communauté épistémique », Sextant [En ligne], 37 | 2020, mis en ligne le 01 novembre 2021, consulté le 24 janvier 2022. URL : http://journals.openedition.org/sextant/338 ; DOI : https://doi.org/10.4000/sextant.338

\section{c) (i) (2)}

La revue Sextant est mise à disposition selon les termes de la Licence Creative Commons Attribution Pas d'Utilisation Commerciale - Partage dans les Mêmes Conditions 4.0 International. 


\title{
La sex research : émergence, développement et éclatement d'une communauté épistémique
}

\author{
Alain GIAMI
}

\section{Introduction : le champ de la sex research}

Le terme de sex research est couramment utilisé dans le champ scientifique pour désigner un paradigme et une communauté épistémique ${ }^{1}$ comprenant des chercheurs, des organisations savantes et des revues qui s'est constituée à partir de la fin des années 1950 aux États-Unis ${ }^{2}$. Ce mouvement s'est largement développé au milieu des années 1960 avant de se diversifier dans un certain nombre de ramifications qui se sont progressivement autonomisées au milieu des années 1990, au point de remettre en question l'hégémonie de la sex research.

Ce constat nous a amené à faire l'hypothèse selon laquelle, loin de représenter la totalité de la recherche scientifique sur la sexualité et face à la difficulté, voire à l'impossibilité, de considérer la sexualité comme un objet scientifique autonome et unifié, la sex research n'aurait représenté dès le départ que la partie la plus visible car très bien organisée - de la recherche scientifique sur la sexualité. Cet article vise ainsi à comprendre à quel moment et dans quels contextes sociaux et historiques est apparue la communauté de la sex research, quels acteurs y sont intervenus, quelle partie de l'ensemble de la recherche scientifique sur la sexualité la sex research a représentée et quels sont les contenus et les thématiques qui s'y sont développés de façon préférentielle. Il vise à explorer l'idée selon laquelle la sex research représente un moment et une forme historiquement située du développement des recherches

${ }^{1}$ M. Meyer et S. Molyneux-Hodgson, « Introduction: The Dynamics of Epistemic Communities ", Sociological Research Online, 15/2, 2011, http://www.socresonline.org.uk /15/2/14.html, 10.5153/sro.

2 J. Gagnon, « Sex research and social change », Archives of Sexual Behavior, 4/2, 1975, p. 111-141. 
scientifiques sur la sexualité dans le monde occidental et principalement aux ÉtatsUnis. Ce moment épistémique a donné lieu à la constitution d'un modèle scientifique généraliste et pluridisciplinaire à vocation internationale avant d'évoluer vers d'autres modèles épistémiques et politiques sous l'effet conjoint de la spécialisation disciplinaire des recherches et la survenue de nouvelles questions sociales et sanitaires dans lesquelles la sexualité, ou certains de ses aspects, en est venue à occuper une place centrale.

Dans un premier temps, l'historiographie de la sex research et sa périodisation seront discutées. Dans un deuxième temps, on présentera les développements de la communauté de la sex research (acteurs, revues, organisations) et l'analyse des sommaires des premiers numéros de revues. Dans un troisième temps, l'éclatement du paradigme et la constitution de nouvelles communautés seront décrits.

\section{Méthode}

Ce travail est fondé sur une analyse de type « narratif $»^{3}$ des sources disponibles dans le champ de la sex research et notamment une analyse des éditoriaux et des sommaires des premiers numéros des revues de la sex research. Il comprend en outre une expérience personnelle de participation à différentes manifestations de cette communauté. Nous avons notamment participé aux conférences de l'International Academy of Sex Research (IASR) à partir de 1994, de la World Association for Sexual Health (WAS) à partir de 19994, de 1'International Society for Sexual Medicine (ISSM) à partir de 2001 ; participé à plusieurs comités de rédaction et comités scientifiques ; évalué des publications scientifiques et last but not least recueilli de nombreux entretiens informels et constitué une photothèque auprès des principaux acteurs intervenant dans ce domaine ${ }^{5}$.

\section{Penser la sex research comme communauté épistémique}

Dans une revue de la littérature consacrée à la notion de « communautés épistémiques », Meyer et al., reprenant les premières définitions de Holzner et Marx ${ }^{6}$, les définissent comme des « communautés de travail pour lesquelles les critères épistémiques occupent la place principale par rapport à d'autres intérêts ou objectifs ». Les membres de telles communautés épistémiques partagent la même foi selon laquelle « les méthodes scientifiques sont le moyen de produire la vérité » ${ }^{7}$. La communauté de la sex research se construit sur la base d'arguments de type scientifique. La dimension

${ }^{3}$ R. FERRARI, « Writing narrative style literature reviews », Medical Writing, 24/4, 2015, p. 230-235.

${ }^{4}$ A. Giami, «Ethnographie d'une conférence médico-scientifique : l'influence de l'industrie pharmaceutique dans le champ de la sexologie », Revue Sociologie / Santé, 30, 2009, p. 187-210.

${ }^{5}$ Le matériel visuel (photographies des principaux acteurs, photographies de scènes collectives durant les congrès, couvertures d'ouvrage) collecté au cours de ce parcours fera l'objet d'une autre publication.

${ }^{6}$ B. Holzner et J. Marx, Knowledge Application: The Knowledge System in Society, Boston, Allyn and Bacon, Inc., 1979, p. 107.

${ }^{7}$ M. Meyer et S. Molyneux-Hodgson, op. cit. 
internationale donnée à ce projet, qui en constitue le versant de stratégie de politique scientifique, est construite comme une nécessité liée à son développement scientifique et au fait de pouvoir réunir une masse critique plus importante de chercheurs dont une partie se trouvent alors en dehors des États-Unis et du Canada et encore plus isolés que les Nord-Américains, dans leurs pays respectifs. On observe donc un double processus avec d'une part l'intention de délimiter de la façon la plus large possible un champ aux frontières mal définies, et de l'autre celle de l'ouvrir au plan international - et principalement au plan européen - en y invitant des chercheurs des différentes disciplines, déjà engagés dans des recherches sur différents aspects de la sexualité : hormonaux, psychologiques ou sociologiques. La sex research se constitue en développant un modèle épistémique et en s'appuyant sur la création de sociétés savantes et de revues et par l'organisation de conférences et séminaires qui permettent de regrouper le réseau de ses membres.

\section{L'historiographie de la sex research : un champ de controverses ?}

L'historiographie de la sex research - que l'on peut qualifier de réflexive - a été développée en grande partie par des auteurs appartenant eux-mêmes à ce champ de recherche, qui ont ainsi contribué à créer cette communauté. Des formes d'egohistoire ont été développées par des personnages tels que le sociologue Ira Reiss $^{8}$, chez qui l'expérience autobiographique scande la trame de l'histoire de la science de la sexualité (sexual science). John Gagnon, qui est l'un des premiers à avoir tenté de conceptualiser la sex research et à lui donner une perspective historique ${ }^{9}$, regroupe, sous le terme de sex research, l'ensemble des recherches sur la sexualité et situe l'origine de celles-ci en Europe occidentale à la fin du XIX ${ }^{\mathrm{e}}$ siècle avant son redéploiement aux États-Unis au début des années 1920 : dans cette optique, la sex research - telle que définie par Gagnon - constitue un domaine général englobant différentes formes d'approches scientifiques de la sexualité (enquêtes, travaux de laboratoire, psychanalyse, anthropologie, histoire, sciences politiques, etc.) qui contribue au « changement social ». La " révolution » occasionnée par la publication des Rapports Kinsey en 1948 et 1953 met en pleine lumière la diversité des comportements et des relations sexuelles, souligne l'importance des relations et des contacts homosexuels et relativise la place du mariage comme principale forme d'organisation sociale de la sexualité ${ }^{10}$. La sex research vise ainsi à participer aux débats et aux critiques de la morale sexuelle puritaine ${ }^{11}$. C'est dans cette perspective qu'à partir du début des années 1960, la sex research s'inscrit de plain-pied dans le

${ }^{8}$ I. ReISS, An Insider's View of Sexual Science since Kinsey, Lanham, Rowman \& Littlefield Inc., 2006.

9 J. GaGnON, op. cit.

10 J. Gagnon, "Gender preferences in erotic relations: The Kinsey scale and sexual scripts ", in D. McWhirter, S. A. SAnders et J. M. Reinisch (dir.), Homosexuality/ Heterosexuality: Concepts of sexual orientation, New York, Oxford University Press, 1990, p. 177-207.

${ }^{11}$ I. Reiss, op. cit. et J. BAnCroft, «Alfred C. Kinsey and the Politics of Sex Research », Annual Review of Sex Research, 15, 2004, p. 1-39. 
mouvement de la modernisation de la sexualité ${ }^{12}$, marqué par un optimisme sexuel, par l'ambition de développer une forme d'objectivité scientifique se situant en dehors des valeurs morales ${ }^{13}$ et par l'entrée de la sexualité dans la conversation publique. Elle prend place dans un ensemble de pratiques culturelles, intellectuelles et artistiques qui précéderont la survenue de la « révolution sexuelle » des années 1960 et $1970^{14}$, aux côtés de la découverte et de la diffusion de la contraception hormonale, de l'identification du " phénomène transsexuel ${ }^{15}$, des travaux de Masters \& Johnson sur les processus psychophysiologiques de l'orgasme ${ }^{16}$ et, quelques années plus tard, la dépathologisation de l'homosexualité ${ }^{17}$. Cette première phase de la sex research a été interprétée par Irvine comme un moment où la production et la diffusion des discours scientifiques contiennent une dimension performative de construction de la diversité de la réalité sociale en matière de sexualité ${ }^{18}$. Cette dimension performative et notamment le projet de normalisation de l'homosexualité a été considérée comme « anti-américaine » par la Commission McCarthy et a valu un certain nombre de difficultés à Kinsey lui-même et aux chercheurs de l'Institut Kinsey ${ }^{19}$.

Quarante ans plus tard, adoptant une autre perspective, Dowsett resitue la sex research dans un contexte plus large dans lequel il distingue la sexologie et les critical studies in sexuality de la sex research, chacun de ces domaines ayant des caractéristiques spécifiques. La sexologie représenterait la partie clinique de cet ensemble face à laquelle la sex research a effectivement tenté de prendre des distances. La sex research serait ancrée selon Dowsett dans la quantification et la mesure de la fréquence des comportements sexuels et ne prendrait en compte ni les significations attribuées à ceux-ci par les acteurs ni les contextes sociaux, politiques et culturels dans lesquels ils se déploient. Les critical studies in sexuality représenteraient ainsi la forme la plus récente de développement des approches scientifiques de la sexualité, notamment dans les sciences sociales, en travaillant sur des populations restreintes et en développant des approches qualitatives et notamment des ethnographies très précises $^{20}$. Dans la perspective critique développée par Dowsett, une étude récente fondée sur une démarche empirique d'analyse du corpus des articles publiés dans le Journal of Sex Research (JSR) entre 2010 et 2015 met en lumière les orientations

${ }^{12}$ P. Robinson, The modernization of sex, New York, Harper \& Row, 1976.

13 J. Irvine, Disorders of Desire. Sexuality in Modern American Sexology, Philadelphia, Temple University Press, 2005.

${ }^{14}$ D. Allyn, Make love not war. The Sexual Revolution: an unfettered history, Boston, Little, Brown \& Company, 2000 ; J. EscoffIER, Sexual Revolution, New York, Thunder's Mouth Press, 2003 ; et G. Hekma et A. Giami (dir.), Sexual Revolutions, Houndmills, Basingstoke, Palgrave, 2014.

${ }^{15}$ H. Benjamin, The Transsexual Phenomenon, New York, Julian Press, 1966.

16 W. Masters et V. Johnson, Human sexual response, Boston, Little, Brown and $\mathrm{C}^{\circ}$, 1966

${ }^{17}$ R. BAYer, Homosexuality and American Psychiatry. The Politics of Diagnosis, New York, Basic Books, 1981.

18 J. IRVINE, op. cit.

19 J. BANCROFT, op. cit.

${ }^{20}$ G. DowsETT, «The price of pulchritude, the cost of concupiscence: how to have sex in late modernity », Culture, Health and Sexuality, 17-Suppl. 1, 2015, p. 5-19. 
politiques de la littérature publiée dans cette revue et notamment son " hétérocis-centrisme » et la focalisation sur les questions du risque et des maladies plutôt que sur une approche positive du plaisir sexuel et en particulier de ses formes non hétérocentrées ${ }^{21}$.

On observe dans le décalage entre les positions de Gagnon et Dowsett toutes les évolutions du domaine de la recherche scientifique sur la sexualité qui ont été occasionnées à trente ans d'intervalle. Alors qu'aux débuts de la sex research on pouvait parler d'une unique communauté dotée de ses revues, de ses sociétés et de ses conférences - certes restreinte à quelques dizaines de personnes et d'un modèle épistémique unifié, mais relativement ouvert et diversifié, il est désormais difficile de maintenir l'unité de ce dispositif. Alors que Gagnon considérait que les recherches psychophysiologiques de Masters \& Johnson faisaient partie intégrante de la sex research au même titre que les recherches de Kinsey ${ }^{22}$, Dowsett situe pleinement les critical sexuality studies comme partie intégrante des sciences sociales et en particulier dans le giron des «critical studies » qui se sont développées sur le genre, les femmes ou sur d'autres communautés de pratiques sexuelles et d'identités de genre. Les approches psychologiques et biomédicales en viennent à constituer l'un des objets d'étude des critical sexuality studies, ce qui est une façon de s'en distancier.

Ces évolutions dans les conceptions de la sex research reflètent les évolutions des conceptions et des scénarios culturels en matière de sexualité et notamment l'abandon de l'intérêt pour les dimensions biopsychologiques longtemps considérées comme soubassement indispensable à l'ensemble de la recherche. Le décalage observé entre ces deux chercheurs pose la question des relations entre les sciences biopsychologiques et les sciences sociales : celles-ci peuvent-elles être pensées comme le soubassement d'un modèle unifié biopsychosocial ou bien deviennent-elles l'un des objets d'étude des sciences sociales et historiques ? Enfin, c'est la nature de l'engagement dans le champ social qui a changé radicalement. Pour la sex research, l'important était de mettre en évidence la diversité des pratiques et des relations sexuelles et ce projet apparaissait comme subversif en soi dans un contexte états-unien marqué par un conformisme puritain. Trente ans plus tard, c'est du point de vue de la diversité sexuelle que l'on analyse la diversité et les modèles dominants.

\section{Les prémices de la sex research : de Berlin à New York}

De nombreux travaux historiques ont documenté les origines de la recherche sur la sexualité : Sexualwissenschaft en Europe, et notamment en Allemagne à la fin du XIX ${ }^{\mathrm{e}}$ siècle $^{23}$. On retrouve dans les origines berlinoises de la Sexualwissenschaft européenne les principales caractéristiques qui vont constituer le modèle de

${ }^{21}$ A. JonES, « Sex is not a problem: The erasure of pleasure in sexual science research », Sexualities, 22/4, 2019, p. 643-668.

${ }^{22}$ De fait, Masters \& Johnson ont été membres de l'IASR et ont participé à plusieurs des congrès de cette organisation.

${ }^{23}$ R. Dose, "The World League for Sexual Reform: Some Possible Approaches », Journal of the History of Sexuality, 12/1, 2003, p. 1-15 ; F. TAMAGNE, « La Ligue mondiale pour la réforme sexuelle : la science au service de l'émancipation sexuelle ? », Clio, 22, 2005, 
communauté épistémique de la sex research: une organisation locale dans un premier temps, comme première étape de la dimension internationale, une revue et une communauté internationale qui se retrouve lors de congrès. Mais alors que l'expérience berlinoise, et notamment celle menée par Hirschfeld, reste en grande partie fondée sur l'engagement politique en faveur des homosexuels, de la diversité sexuelle et des personnes transgenres et intersexuées et la constitution d'organisations alternatives, on ne va pas retrouver les mêmes préoccupations dans les développements qui auront lieu aux États-Unis.

La sex research nord-américaine abandonne, dans un premier temps, le lien intrinsèque avec la question de l'émancipation sociale et politique pour s'inscrire dans des préoccupations de santé publique, d'eugénisme, de morale publique, d'hygiène sociale et de recherche fondamentale sur un modèle académique et se développe grâce à l'appui des grandes fondations industrielles et notamment la Fondation Rockefeller, dans un premier temps, et, plus tard la Fondation Ford ${ }^{24}$. Les périodes allemandes et la première époque nord-américaine ont cependant en commun de construire des dispositifs conceptuels qui prennent appui sur des dispositifs organisationnels.

\section{Première étape : l'institutionnalisation de la recherche sur les « problèmes liés à la sexualité » aux États-Unis}

Le Comité pour la recherche sur les problèmes de la sexualité (Committee for Research in Problems of Sex, CRPS), créé en 1922, est institué au sein du National Research Council's Division of Medical Sciences (Conseil national de la recherche Division des sciences médicales) avec la coopération du Bureau national d'hygiène sociale. Il est financé par la Fondation Rockefeller ${ }^{25}$ et constitue le point de départ d'une communauté épistémique hybride qui englobe des acteurs scientifiques, mais aussi des entrepreneurs (administrateurs et fonctionnaires publics). L'objectif de ce comité est de développer la recherche sur ce qui, à cette époque, est considéré comme les questions fondamentales liées à la sexualité telles que la génétique, la détermination des caractères sexuels, le développement sexuel de l'enfant, les hormones sexuelles, le problème des relations interpersonnelles, les fonctions sexuelles et reproductives, la différenciation sexuelle chez les animaux et les plantes $^{26}$. Les premiers membres du Comité appartiennent notamment aux domaines

p. 101-121; H. OOSTERHUIS, Stepchildren of nature. Krafft-Ebing, psychiatry and the making of sexual identity, Chicago, The University of Chicago Press, 2000.

${ }^{24}$ D. Di Mauro, Sexuality Research in the United States. An Assessment of the Social and Behavioral Sciences, New York, Social Sciences Research Council, 1995.

${ }^{25}$ V. L. Bullough, "The Rockefellers and Sex Research », The Journal of Sex Research, 21-2, 1985, p. 113-125 ; V. L. Bullough, « Katharine Bement Davis: Sex research and the Rockefeller Foundation », Bulletin of the History of Medicine, 62/1, 1988, p. 74-89.

${ }^{26}$ Liste établie par Frank Lillie en 1922 dans S. D. ABERLE et G.W. Corner, Twenty-Five Years of Sex Research: History of the National Research Council Committee for Research in Problems of Sex 1922-1947, Philadelphia, PA, W.B. Saunders Company, 1953. 
de la biologie, de la physiologie, de la psychologie, de la psychopathologie et dans une moindre mesure de la sociologie ${ }^{27}$.

$\mathrm{Au}$ cours des vingt premières années de son existence, le CRPS soutient notamment la découverte des premières hormones estrogènes. Mais, paradoxalement, les travaux plus connus, développés avec le soutien du CRPS, sont ceux de l'équipe dirigée par Alfred Kinsey, qui commence à travailler dans le cadre du CRPS dès 1942 et publie les deux rapports sur le comportement sexuel de l'homme et de la femme en 1948 et $1953^{28}$.

Ce comité représente une forme d'institutionnalisation de la recherche sur « certains problèmes liés à la sexualité ». Il inclut un mélange de recherches fondamentales en endocrinologie, de recherches psychosociales et d'études sur les comportements sexuels. Les financements et les appels d'offres proposés renseignent sur ce qui est considéré comme prioritaire et constitutif de ces « problèmes liés à la sexualité », mais aussi sur les domaines qui restent exclus du champ de l'investigation scientifique. On note ainsi une prédominance et une priorité accordée dans un premier temps aux aspects biologiques et surtout hormonaux de la vie sexuelle et reproductive, avec des incursions dans le champ de la psychologie ${ }^{29}$. Dans un deuxième temps, on observe l'émergence d'Alfred Kinsey, zoologiste travaillant sur les comportements sexuels qui va occuper une place de plus en plus importante dans cette communauté ${ }^{30}$.

Kinsey condense toutes les dimensions des enjeux qui se sont joués dans le cadre de ce comité. Il représente une sorte de synthèse entre la biologie, la zoologie et l'approche sur les humains qui fait qu'il va finir par recevoir la quasi-totalité des financements disponibles à partir du moment où la Fondation Rockefeller prend le contrôle du Comité. Il y a ainsi concurrence d'intérêts entre les tenants de la biologie de la reproduction qui refusent de traiter les aspects humains de la sexualité - sujet tabou - et les sciences de la sexualité en dehors de ses aspects reproductifs (scientific research on sexuality) qui apparaissent sulfureuses et considérées comme faisant partie du domaine de la morale plus que de celui de la science. La recherche scientifique sur la sexualité se voit alors encadrée par des organismes publics gouvernementaux et par la Fondation Rockefeller pour répondre à une commande sociale. En dépit de cet encadrement, les recherches sur la sexualité et les chercheurs qui y contribuent restent la cible d'attaques et d'oppositions importantes, et notamment de la part des milieux qui gravitent autour du maccarthysme. C'est sous la pression de ces milieux puissants et organisés que les financements de Kinsey seront ainsi suspendus et que le Comité cessera son activité en $1963^{31}$.

${ }^{27}$ Les archives du Comité sont accessibles sur le site http://www.nasonline.org/about-nas/ history/archives/collections/crps-1920-1965.html.

${ }^{28}$ J. Gagnon, "Gender preferences in erotic relations: The Kinsey scale and sexual scripts », op. cit. ; A. GiAmI, «De Kinsey au sida : l'évolution de la construction du comportement sexuel dans les enquêtes quantitatives », Sciences sociales et santé, IX/4, 1991, p. 23-56.

${ }^{29}$ P. Hegarty, « Beyond Kinsey: The Committee for Research in Problems of Sex and American Psychology », History of Psychology, 15/3, 2012, p. 197-200.

30 J. Gagnon, " Gender preferences in erotic relations: The Kinsey scale and sexual scripts », op. cit.

${ }^{31}$ S. D. Aberle et G. W. Corner, op. cit. 


\section{Deuxième étape : sex research. Paradigme et communauté ?}

La sex research commence à se constituer comme champ de recherche autonome à partir de la fin des années 1950. Les principaux acteurs de ce champ prennent des distances avec les « recherches sur les problèmes liés à la sexualité » pour se consacrer de façon fondamentale aux recherches sur les comportements sexuels et les aspects non reproductifs de la sexualité et leurs déterminants hormonaux et psychologiques, domaines qui avaient été négligés volontairement par le Comité. Les travaux entrant dans ce domaine apparaissent sous différentes dénominations telles que sex research, scientific study of sex ou encore sexual science avec des acceptions différentes selon les moments, les acteurs et les institutions qui les véhiculent. Mais le terme de sex research va s'imposer et se développer grâce à des initiatives individuelles et privées et, au départ, sans le soutien institutionnel d'une organisation ou d'une fondation en dehors de l'appartenance académique de certains de ses membres. Il y a donc une certaine rupture avec le modèle d'une recherche pilotée par des agences publiques soutenue par une fondation au profit d'une recherche présentée comme désintéressée.

On observe en premier lieu une volonté stratégique de réunir dans un même ensemble une communauté de chercheurs travaillant dans des disciplines scientifiques différentes sur un même thème : la sexualité. Cette volonté est fondée sur le constat de la dispersion des travaux de ces chercheurs dans des supports pas souvent réceptifs aux questions de sexualité. Elle vise à créer une communauté entre les chercheurs dispersés dans différents champs de recherche à travers le monde et à se doter de revues scientifiques de haut niveau pour réunir les travaux publiés dans ce domaine, principalement en langue anglaise. La volonté internationaliste est présente dès les débuts de la sex research comme communauté épistémique qui se construit à partir d'une thématique de recherche : la sexualité, aux contours flous et avec l'ambition de créer un champ pluridisciplinaire à partir d'un objet et des questions qu'il pose dans le champ social et dans différentes disciplines scientifiques. Il est important de noter que dès ses débuts, la sex research privilégie la " recherche fondamentale ". Même si certains des principaux acteurs de la sex research ont aussi participé au renouveau de la sexologie et notamment de la sexologie clinique dans le sillon de Masters \& Johnson, au début des années 1960, ces deux courants se sont développés de façon parallèle avec leurs propres congrès, leurs organisations et leurs revues. Quelques chevauchements ont cependant eu lieu au niveau des revues qui ont publié principalement des articles de recherche fondamentale et parfois ouvert leurs colonnes à des articles inscrits dans la pratique clinique sexologique et psychiatrique (les troubles sexuels et les questions transgenres principalement). Il n'y a pas eu de séparation stricte entre les domaines de la sexologie et de la sex research, mais plutôt une forme de porosité entre ces deux domaines avec les cliniciens se regroupant de préférence sous l'étendard de la sexologie et les chercheurs universitaires sous celui de la sex research. La recherche dans ce domaine n'est plus organisée sous l'égide d'une fondation ou des pouvoirs publics qui peuvent lancer des appels d'offres ou distribuer des financements spécifiques pour des projets ou des programmes. Il s'agit désormais pleinement de la constitution de communautés épistémiques fondées sur un intérêt scientifique revendiqué comme tel pour ce qui est considéré alors comme l'ensemble des questions relevant du champ de la sexualité. 


\section{La fondation des premières organisations savantes}

La pluridisciplinarité et l'ambition internationale ont été promues dès la fondation de la première société savante en 1957, la Society for the Scientific Study of Sexuality (SSSS, 4S), et dans les premiers éditoriaux des principales revues qui comportent le terme de sex research dans leur titre : Advances in Sex Research sous la direction de Hugo Belgel en 1963 et Journal of Sex Research sous la direction de Hans Lehfeldt en 1965. La revue Archives of Sexual Behavior est créée, pour sa part, par Richard Green en 1971, puis l'International Academy of Sex Research en 1975 sous la houlette du même Richard Green. Ces organisations et les revues dans lesquelles leurs membres commencent à publier abondamment constituent le noyau dur de la communauté épistémique de la sex research qui s'organise progressivement à partir de cette époque.

Parallèlement à l'essor de la sex research et des deux principales sociétés qui se mettent en place de façon systématique (Academy et 4S), la World Association for Sexology (WAS) est créée en 1978 en développant un modèle organisationnel différent. La WAS constitue dès le départ une fédération de sociétés scientifiques et médicales nationales, de fédérations régionales, de congrès et de revues, regroupant principalement des cliniciens et des éducateurs alors que l'Academy et la 4S sont des sociétés qui proposent une adhésion à des individus sur la base de leur intérêt pour la recherche dans ce domaine, leurs qualifications académiques et la liste de leurs publications et soutenus par un réseau de parrainage. Les principaux fondateurs de la sex research tels que Richard Green et Hans Lehfeldt apportent leur soutien à la WAS avec leur présence active lors de l'organisation des premiers congrès de sexologie médicale en 1974 (Paris), 1976 (Montréal) et 1978 (Rome).

La création de ces différentes communautés épistémiques se situe dans le sillage du mouvement culturel de la « révolution sexuelle » et nous rappelle l'importante composante scientifique de celle-ci ${ }^{32}$. La question politique se pose différemment dans ce contexte : c'est le fait même de parler publiquement de la sexualité dans une optique non reproductive et non conjugale, d'assurer la reconnaissance de la diversité sexuelle et du caractère non pathologique de l'homosexualité qui constitue l'acte libérateur en lui-même, et la pratique de la recherche et de la publication constitue l'essence même de ce geste libérateur. Contrairement aux positions européennes de la fin du XIX ${ }^{\mathrm{e}}$ siècle où le travail scientifique sur la sexualité et la libération sexuelle avaient une visée explicite et des engagements dans des organisations en vue de l'émancipation sociale, la liberté de la recherche sur la sexualité et de façon plus générale la légitimité de la curiosité sexuelle ainsi que le développement d'approches laïques de la sexualité deviennent les composantes centrales de la libération sexuelle et sa principale finalité. À titre d'anecdote significative, Richard Green rapporte qu'il a bénéficié du soutien de Hugh Hefner, le patron de Playboy Magazine qui a aidé - gratuitement avec les services juridiques du magazine - l'Academy à rédiger ses statuts de société « sans profit », selon le droit des affaires des États-Unis, et soutenu la tenue du premier congrès de l'International Academy of Sex Research ${ }^{33}$.

32 J. Escoffier, op. cit.; et D. Allyn, op. cit.

33 R. Green, «Hugh Hefner, the International Academy of Sex Research, and Its Founding President », Archives of Sexual Behavior, 46, 2017, p. 2211-2212. 


\section{Les éditoriaux des revues}

L'analyse des éditoriaux publiés dans les premiers numéros des deux revues fondatrices - qui sont des documents stratégiques bien plus que des documents scientifiques stricto sensu - met en évidence que les rédacteurs en chef de celles-ci ont bien le projet de construire une communauté épistémique fondée sur un modèle qui vise à rassembler les différentes disciplines de la façon la plus large possible. Dans l'éditorial d'Advances in Sex Research, Hugo Belgel propose un programme multidisciplinaire qui pourra s'étendre sur plusieurs années.

The area of sex research extends into many fields-to name only a few examples, into the fields of psychiatry, psychology, zoology, anthropology, religion, philosophy, demography, legislation, and various branches of medicine; it involves customs and courtships, need satisfaction and its social regulations, fertility and infertility, pleasure and ethics, child rearing and education, and individual behavior, not only that which is regarded as sexually normal or sexually aberrant but also much of that which on the surface appears to be unrelated to sexual influences. No single volume can do justice to this abundance. In its totality and over the years, it is anticipated that this series will cover the whole field of $\operatorname{sex}^{34}$.

Dans le premier éditorial de sa revue Archives of Sexual Behavior, Richard Green tient un discours similaire en indiquant la volonté de rassembler au-delà des barrières les disciplines biomédicales, psychologiques et des sciences sociales. En outre, Green inclut les universitaires aussi bien que les cliniciens qui interviennent sur les questions sexuelles. Il a l'ambition notamment de réunir des psychanalystes aussi bien que des psychologues cognitivistes. Mais surtout, Green affirme une volonté internationaliste plus marquée et insiste pour sa part sur le haut niveau académique qui est attendu des travaux publiés dans Archives.

The Archives of Sexual Behavior hopes to be the first journal to collate effectively research contributions of consistently high caliber from scholars throughout the world. It hopes to incorporate, among others, the research strategies of the clinician, includ- ing the psychoanalyst and behavior therapist, with the perspective of the social scientist and the findings of the laboratory investigator ${ }^{35}$.

${ }^{34}$ H. G. Belgel, «Preface », Advances in Sex Research, 1, 1963, p. 14-15.

« La recherche sur la sexualité s'étend à de nombreux domaines - pour ne citer que quelques exemples - dans les domaines de la psychiatrie, de la psychologie, de la zoologie, de l'anthropologie, de la religion, de la philosophie, de la démographie, du droit et de diverses branches de la médecine ; elle concerne les coutumes et les rituels de cour, la satisfaction des besoins et sa réglementation sociale, la fertilité et l'infertilité, le plaisir et l'éthique, l'éducation des enfants et le comportement individuel, non seulement ce qui est considéré comme sexuellement normal ou aberrant, mais aussi une grande partie de ce qui, à première vue, semble sans rapport avec les influences sexuelles. Aucun volume ne peut à lui seul rendre justice à cette abondance. Dans sa totalité et au fil des ans, il est prévu que cette série couvre l'ensemble du domaine de la sexualité » (traduction de l'auteur).

35 R. GREen, «A statement of purpose », Archives of Sexual Behavior, 1/1, 1971, p. I.

« Les Archives of Sexual Behavior espèrent être la première revue à rassembler des contributions de chercheurs de haut niveau issus du monde entier. La revue a l'ambition d'intégrer, entre autres, les méthodes de recherche des cliniciens, en ce compris des psychanalystes et des 


\section{Les sommaires des premiers numéros}

Encadré 1 : The Journal of Sex Research, vol. 1, nº 1, mars 1965

The Sex Instinct and Human Eroticism / John Money

Sex Education of the Young Child / Lili Peller

Principles of Children's Gynecology in Czechoslovakia / Rudolf Peter

Legal Abortions in Romania / Karl Heinz Mehlan

Psychopathology of Transvestism and Transsexualism / Johann M. Burchard

Group Psychotherapy with Sexually Deviant Offenders (Pedophilies): The Peer Group as an Instrument of Mutual Control / Valdemar Hartman

Encadré 2 : Archives of Sexual Behavior, vol. 1, n 1, 1971

\section{A statement of purpose / Richard Green M.D}

Experience with pornography: Rapists, pedophiles, homosexuals, transsexuals, and controls / Michael Goldstein Ph.D., Harold Kant LL.B., Lewis Judd M.D....

The bisexual identity of transsexuals: Two case examples / Robert J. Stoller M.D., Lawrence

E. Newman M.D.

Lower-class sexuality: Some emotional and social aspects in West German males and females / Volkmar Sigusch M.D., Gunter Schmidt Ph.D.

Psychosexual development, maternalism, nonpromiscuity, and body image in 15 females with precocious puberty / John Money Ph.D., Paul A. Walker B.S.

The role of gonadal hormones in the sexual behavior of the rhesus monkey and human: A literature survey / William G. Luttge Ph.D.

Hypersexuality, anti-androgens, and testicular function / F. Ott

Male transsexualism: An endocrine study / M. Philbert

On peut observer, dès la publication des premiers numéros de ces deux revues, un intérêt pour les aspects biologiques, hormonaux, psychologiques et sociologiques ainsi qu'un fort intérêt pour les questions de l'identité de genre et du transsexualisme. Le psychologue John Money, spécialiste des questions de l'identité de genre et des paraphilies, apparaît dans les sommaires de ces deux premiers numéros. Par ailleurs, Archives publie un article entrant dans le champ de la sexologie animale. Les enquêtes en population coexistent avec les travaux de laboratoire, les recherches cliniques ou éducatives ainsi que les études de cas. Un psychanalyste (Stoller), qui a par ailleurs décliné l'invitation à entrer dans le club très sélect de l'Academy, apparaît en compagnie d'un spécialiste des thérapies comportementales. Les deux revues font preuve d'ouverture internationale avec, dans le JSR, deux articles - sociologiques - en provenance de Roumanie et de Tchécoslovaquie, alors qu'Archives commence un partenariat avec une équipe de psychiatres et sociologues allemands de Hambourg (RFA).

Les sommaires de ces deux revues sont situés dans une continuité remarquable avec le programme de recherche développé dans le modèle américain du Comité de

thérapeutes comportementalistes, avec la mise en perspective par les recherches en sciences sociales et les résultats de la recherche empirique » (traduction de la rédaction). 
1921. On y observe le même intérêt pour la recherche fondamentale (et clinique) sur les questions hormonales et celles liées aux identités de genre. Les principales innovations de la sex research se situent du côté du développement des approches psychosexuelles et des thérapies qui se développeront au cours des années 1970 et des travaux sociologiques internationaux.

Près de quinze années après la fondation de la revue et de l'International Academy of Sex Research, son fondateur Richard Green dresse un bilan de l'œuvre accomplie dans ce domaine ${ }^{36}$. La volonté de créer une communauté épistémique, débarrassée des contingences commerciales et de la nécessité de trouver un équilibre financier apparaît tout à fait présente. Green en profite pour se distinguer du JSR et de la $4 \mathrm{~S}$ à qui il reproche de s'être engagée dans le domaine de l'éducation et de la pratique clinique au détriment de la recherche scientifique fondamentale :

As the science of sex research grew, its subdisciplines included clinical outcome studies assessing diverse strategies of sex therapy, sexual physiology with penile and vaginal measures of erotic arousal, and developmental studies of persons occupying scattered points on the sexual identity spectrum, transsexuals, transvestites, homosexuals. For other researchers working with non-humans, rats bumped, gorillas humped, kangaroos jumped, and neurotransmitters pumped.

We were all pursuing the hidden knowledge of our new science. But we were members of too diverse a group of "home" disciplines and of isolated professional organizations. There was no common meeting ground for exchange between the relatively few scientists, world-wide, whose exclusive professional commitment was sex research. There was no special opportunity for sharing, either in person or on the printed page. The first remedy was the establishment of Archives of Sexual Behavior. The second was the establishment of the International Academy of Sex Research ${ }^{37}$.

${ }^{36}$ Dans son étude sur l'histoire de la sex research aux États-Unis entre 1900 et 1990, Vern Bullough ne fait aucune référence ni à l'International Academy of Sex Research ni à Richard Green, qui avait pourtant fait des études de médecine. V. Bullough, « American Physicians and Sex Research and Expertise, 1900-1990 », Journal of the History of Medicine and Allied Sciences, 52/2, 1997, p. 236-253.

${ }^{37}$ R. GREEN, «The International Academy of Sex. In the Beginning », Archives of Sexual Behavior, 14/4, 1985, p. 294.

«Au fur et à mesure que la sex research s'est développée, ses sous-disciplines ont inclus des études cliniques évaluant diverses stratégies de thérapie sexuelle, la physiologie sexuelle avec des mesures péniennes et vaginales de l'excitation érotique, et des études de développement de personnes occupant des points dispersés sur le continuum de l'identité sexuelle, transsexuels, travestis, homosexuels. Pour d'autres chercheurs travaillant avec des non-humains, les rats se sont cognés contre des parois, les gorilles ont fait des bonds, les kangourous ont sauté et les neurotransmetteurs ont été pompés.

Nous étions tous à la recherche du savoir caché de notre nouvelle science. Mais nous étions membres d'un groupe trop diversifié de disciplines et d'organisations professionnelles isolées. Il n'y avait pas de lieu de rencontre commun pour les échanges entre des scientifiques relativement peu nombreux, dans le monde entier, dont l'engagement professionnel exclusif était la sex research. Il n'y avait pas d'occasion particulière de partage, que ce soit en personne ou à travers des revues. Le premier remède à cette situation a été la création des Archives of Sexual Behavior. Le second a été la création de l'International Academy of Sex Research » (traduction de l'auteur). 
Dans cet article, Green donne en outre la liste des 53 personnes qui ont été cooptées comme membres de l'Academy en 1973 et rappelle que les statuts de l'Academy ont fixé à 250 personnes le nombre maximum de membres. La volonté internationaliste apparaît dans le propos, mais dans la pratique, on observe que la grande majorité des articles publiés dans les deux revues ont été produits aux États-Unis ou au Canada et que les collaborations internationales pour une même étude ne sont pas très nombreuses. Après en avoir été le fondateur et le premier président, Richard Green propose la présidence de l'Academy au Britannique John Bancroft - un psychiatre et spécialiste de médecine de la reproduction -, qui deviendra en 1995 le directeur du Kinsey Institute à Bloomington (Indiana). Green se félicite aussi de la tenue du deuxième congrès de l'Academy à Hambourg (RFA) sous la direction de Gunther Schmidt, un psychiatre et chercheur allemand qui sera élu, à son tour, président de l'Academy en 1981. La présidence de Gunther Schmidt marquera le début de l'influence du groupe allemandaméricain (Anke Ehrhardt, Heino Meyer-Bahlburg, Gotz Kockott, Ulrich Clement) qui emportera la présidence de l'Academy à cinq reprises entre 1981 et 2001, soit l'un des plus forts contingents non américains (USA).

\section{Troisième étape : segmentation et spécialisation de la recherche scientifique sur la sexualité. L'éclatement de la sex research}

Entre le début des années 1960 et le milieu des années 1990, le champ de la recherche scientifique sur la sexualité connaît un accroissement très important. Dans son éditorial d'Archives of Sexual Behavior (2002), Ken Zucker, qui a repris le flambeau de cette revue à Richard Green, ne recense pas moins de soixante-seize revues situées dans le champ lato sensu des recherches sur la sexualité et des études de genre qui ont fait leur apparition ${ }^{38}$. Alors que les premières revues des années 1960 se sont d'emblée inscrites dans une perspective multidisciplinaire et multithématique, les revues qui paraissent à partir du milieu des années 1970 font apparaître une forte tendance à la spécialisation et à la réduction de leur périmètre épistémique. Cette spécialisation se développe dans deux directions. Il s'agit d'une part d'une spécialisation fondée sur l'autonomie et l'identité des différentes disciplines (psychologie, sociologie, cultural studies, anthropologie, médecine sexuelle, éducation sexuelle, etc.) qui en viennent à développer leurs propres programmes de recherche disciplinaires, et d'autre part de la constitution de thèmes de recherche en disciplines et par conséquent en revues spécialisées. On assiste ainsi à l'éclosion et à la spécialisation des thématiques et des champs disciplinaires associés au genre, aux approches féministes et gay, à la question du VIH-sida, à la médecine sexuelle, aux différentes formes de thérapies, à l'éducation sexuelle, aux questions trans, etc. Zucker lui-même semble relativiser l'usage du terme de sex research et parle de la « renaissance de la sexologie » comme pour montrer que les choses ont changé.

À plusieurs reprises, le développement de nouveaux domaines de recherche plus spécialisés dans un champ ou dans une discipline est formulé comme une rupture avec le paradigme et la communauté de la sex research. Ce modèle est désormais perçu comme

${ }^{38}$ K. ZuCKER, «From the Editor's Desk: Receiving the torch in the Era of Sexology Renaissance », Archives of Sexual Behavior, 31/1, 2002, p. 1-6. 
ayant un périmètre épistémique beaucoup plus réduit que celui auquel ses principaux promoteurs et défenseurs prétendaient encore dans un passé relativement récent. Ainsi, au lieu d'incarner l'ensemble du champ des recherches scientifiques sur la sexualité, la sex research en vient à ne plus représenter qu'une de ses multiples formes de spécialisation. Les développements de nouveaux domaines et les ruptures d'avec la sex research viennent de différents côtés. Et de chacun de ces côtés, le besoin de nouvelles approches méthodologiques est exprimé pour combler les limites de la sex research.

\section{Sciences sociales et santé publique}

Tout d'abord, le rapport établi par Diane di Mauro en $1995^{39}$ constitue un événement majeur, car il émane d'un programme de recherche financé par la Fondation Ford. Ce rapport paraît dans un contexte marqué par le développement des grandes enquêtes nationales sur les comportements sexuels visant à la réponse publique à l'épidémie de VIH-sida et par les controverses qui se développent à propos du financement de l'enquête nationale menée aux États-Unis, qui dévoile les enjeux politiques qui se déchaînent autour de la connaissance scientifique sur la sexualité ${ }^{40}$. L'épidémie de sida va susciter un nombre incalculable de travaux d'enquête visant à identifier les pratiques sexuelles à risque des « groupes à risque » ${ }^{41}$. Di Mauro témoigne du double mouvement de rupture et de continuité qui anime le développement de ces nouvelles recherches.

Sexuality research today represents the continuation of a long tradition of primarily individual scholarship on the topic. Occurring most often within a clinical or academic setting, what is specifically identified as sexuality research is that which focuses on sexual physiology, anatomy and therapeutic issues, rather that research that addresses the social, cultural or behavioral topics of sexuality. Social and behavioral research on sexuality is often embedded within the larger research questions in the range of social sciences disciplines, including sociology, psychology, anthropology and history. Sexuality topics are also being addressed by researchers in education, biology, medicine, and public health, again integrated within larger issues researched by each discipline. Very little of this research has sexuality as its primary focus, and that which does is mostly limited to small population samples with a very narrow focus on specific behaviors, within the framework of the discipline ${ }^{42}$.

${ }^{39}$ D. DI MAURo, op. cit.

40 E. Laumann, J. Gagnon, R. Michael et S. Michaels, « A political history of the National Sex Survey of Adults », Family Planning Perspectives, 261, 1994, p. 34-38.

${ }^{41}$ J. Y. Catania, J. T. Moskowitz, M. Ruiz et J. Cleland, « A review of national AIDSrelated behavioral surveys », AIDS, 10-Suppl. A, 1996, p. 183-190 ; A. Giami et M.-A. SCHILTZ, « Representations of Sexuality and relations between partners: Sex research in France in the era of AIDS », Annual Review of Sex Research, 7, 1996, p. 125-157.

42 D. DI MAURO, op. cit.

«La recherche sur la sexualité représente aujourd'hui la continuation d'une longue tradition d'études essentiellement individuelles sur le sujet. Se déroulant le plus souvent dans un cadre clinique ou universitaire, la recherche sur la sexualité est spécifiquement axée sur la physiologie sexuelle, l'anatomie et les questions thérapeutiques, plutôt que sur les aspects sociaux, culturels ou comportementaux de la sexualité. La recherche sociale et comportementale sur la sexualité s'inscrit souvent dans le cadre de questions de recherche plus larges dans l'ensemble des disciplines des sciences sociales, notamment la sociologie, la psychologie, l'anthropologie et 
On note ici le déplacement de la sex research vers la sexuality research qui passe sous silence tous les travaux de type sociologique, anthropologique ou même historique qui ont constitué une part importante de la sex research depuis ses débuts tant au niveau des thématiques que de la présence de chercheurs spécialisés, pour n'en retenir que les recherches psychologiques et cliniques qui ignoreraient les aspects sociaux et politiques associés à la sexualité. Le rejet de la sex research représente aussi la rupture avec la première génération des pionniers. Le rapport de di Mauro commandité par le Social Science Research Council, une organisation financée pendant de nombreuses années par la Fondation Ford, a une valeur programmatique et un modèle de fonctionnement équivalent à celui du défunt Committee for Research in Problems of Sex, autrefois financé par la Fondation Rockefeller. Il est composé d'un comité qui a pour mission de financer des programmes de recherche, des séminaires et des bourses d'études en vue de la réalisation de doctorats dans le domaine des sciences sociales sur les questions de sexualité. Dans le cadre de ce comité, on observe le déclin de l'intérêt scientifique pour les questions biomédicales et cliniques (hormones, physiologie, pathologies). Le rapport de di Mauro appelle à la constitution d'un nouveau modèle sur la base du constat de la carence de travaux dans le domaine des sciences sociales et des enquêtes menées auprès de grands échantillons de la population générale. L'intérêt est désormais porté sur les comportements sexuels, le contexte de la santé publique, les approches anthropologiques et les droits humains et les discriminations à l'égard des groupes LGBT. Les grandes enquêtes en population vont coexister avec les travaux de type ethnographique.

\section{Les critical sexuality studies}

Le programme proposé par le Social Science Research Council de la Fondation Ford préfigure le développement de revues résolument inscrites dans le champ des sciences sociales et porteuses d'une dimension critique aussi bien à l'encontre de la sex research que du fonctionnement social et de la place réservée à la sexualité et aux questions de genre et d'orientation sexuelle dans le monde anglo-américain. Ces développements se sont amplifiés avec la création de nouvelles revues telles que Sexualities (1998), Culture, Health and Sexuality (1999) dans le domaine des critical sexuality studies. La publication en 2004 du premier numéro de la revue Sexuality Research and Social Policy ${ }^{43}$ inaugure l'intérêt pour les droits humains, la santé publique et les alliances avec les activistes.

Le terme de critical sexuality studies est forgé par Kenneth Plummer, le premier éditeur en chef de la revue Sexualities dont le premier numéro paraît en 1998 en Grande-Bretagne.

l'histoire. Les sujets liés à la sexualité sont également abordés par les chercheurs en éducation, en biologie, en médecine et en santé publique, là encore intégrés dans les questions plus larges étudiées par chaque discipline. Très peu de ces recherches ont pour objet principal la sexualité, et celles qui l'ont fait se limitent pour la plupart à de petits échantillons de population, avec un accent très étroit sur des comportements spécifiques, dans le cadre de la discipline » (traduction de l'auteur).

${ }^{43}$ G. Herdt, «A welcome from the editor », Sexuality Research and Social Policy, 1/1, 2004, p. 1-6. 
We are hoping that the journal will not duplicate any other in the field far; though it will take a little while to establish its own clear terrain. Our prime aim is to provide a critical, international, social science / cultural studies-based journal, which focuses upon the shifting nature of human sexualities in societies. The disciplines which it should serve will primarily be sociology, social psychology, ethnographic and qualitative research, cultural history and cultural anthropology, cultural studies, social geography, feminist research, lesbian and gay studies, and media studies. It will be international in scope, though with an emphasis on English speaking cultures. And it is a hope that, in due time, Sexualities will become the major academic journal to analyse the cultural and social nature of human sexualities ${ }^{44}$.

Les critical sexuality studies proposent, à leur tour, de ratisser large en englobant l'ensemble des nouveaux courants de recherche remettant en cause les modèles dits essentialistes de la sexualité et en épousant les différentes formes de studies qui commencent à se développer en créant de nouvelles sous-disciplines à partir des thématiques et des intérêts de groupes minoritaires et du féminisme.

Se situant dans la même perspective que celle des critical sexuality studies, la revue Culture, Health and Sexuality propose dès 1999, sous la direction de Peter Aggleton, une approche plus centrée sur les questions de santé, les questions d'intervention éducative et les politiques de santé et de droits humains.

It is in the interface between sexual meanings and beliefs, sexual cultures and practices, and sexual identities and positioning as they relate to health, that this journal intends to make its special mark. Culture, Health and Sexuality will offer a lead international forum for an exploration of such concerns along with their policy and practice implications ${ }^{45}$.

Les auteurs les plus impliqués dans le domaine de la santé et issus pour la plupart d'entre eux du milieu des chercheurs très tôt engagés dans la recherche sur le sida vont se retrouver dans une revue plus spécialement centrée sur les aspects de santé

${ }^{44}$ K. Plummer, «Introducing sexualities (editorial) », Sexualities, 1/1, 1998, p. 5-10.

« Nous espérons que la revue ne fera pas double emploi avec d'autres revues dans ce domaine, même s'il faudra un peu de temps pour définir son propre terrain. Notre objectif premier est de réaliser une revue critique, internationale, basée sur les sciences sociales et les études culturelles, qui se concentre sur les modifications des sexualités humaines dans les sociétés. Les disciplines qu'elle devrait servir seront principalement la sociologie, la psychologie sociale, la recherche ethnographique et qualitative, l'histoire culturelle et l'anthropologie culturelle, les études culturelles, la géographie sociale, la recherche féministe, les études sur les lesbiennes et les gays, et les études sur les médias. Elle aura une portée internationale, mais l'accent sera mis sur les cultures anglophones. Et il est à espérer qu'en temps voulu, Sexualities deviendra la principale revue universitaire à analyser les dimensions culturelle et sociale des sexualités humaines » (traduction de l'auteur).

45 P. Aggleton, S. Kippax, P. Mane, R. Parker et B. De Zalduondo, « Editorial », Culture, Health and Sexuality, 1/1, 1999, p. 1-2.

"C'est dans l'interface entre les significations et les croyances sexuelles, les cultures et les pratiques sexuelles, et les identités et le positionnement sexuels en rapport avec la santé, que cette revue entend laisser sa marque particulière. Culture, Health and Sexuality offrira un forum international de premier plan pour l'exploration de ces préoccupations ainsi que de leurs implications politiques et pratiques » (traduction de l'auteur). 
publique, de politiques publiques et de droits humains, Sexuality Research and Social Policy, qui est aussi financée par le programme de la Fondation Ford. L'éditorial de Gilbert Herdt, un anthropologue qui a produit des travaux importants sur la vie érotique des populations de Papouasie-Nouvelle-Guinée, dessine les nouvelles perspectives de recherche :

Sexuality Research and Social Policy: Journal of NSRC is a multi-disciplinary, online peer-reviewed journal that publishes state-of-the-art empirical research on sexuality, theoretical and methodological discussions, and the implications of this evidence for U.S. and international policies regarding sexual health, sexuality education, and sexual rights in diverse communities ${ }^{46}$.

Toutes les revues qui s'inscrivent dans ce courant des critical sexuality studies revendiquent la centralité des sciences sociales et des approches qualitatives et à l'instar des principales revues de la sex research, une dimension internationale désormais beaucoup plus ouverte aux perspectives postcoloniales. Une grande partie des chercheurs engagés dans cette perspective se retrouveront dans le cadre de l'International Association for the Study of Sexuality, Culture and Society (IASSCS) fondée en 1997 à Amsterdam et qui sera dissoute en 2018 à la suite de conflits de gouvernance interne.

\section{L'essor de la médecine sexuelle}

L'essor de la médecine sexuelle ${ }^{47}$ représente l'autre versant de l'expression critique et de la rupture avec la sex research. Son développement s'inscrit indubitablement dans le contexte de la pharmacologisation et de la médicalisation de la sexualité et bénéficie du soutien de l'industrie pharmaceutique ${ }^{48}$. La création de l'International Society for

\section{${ }^{46}$ G. HERDT, op. cit.}

"Sexuality Research and Social Policy: Journal of NSRC est une revue multidisciplinaire en ligne, évaluée par des pairs, qui publie des recherches empiriques de pointe sur la sexualité, des discussions théoriques et méthodologiques, et les implications de ces données pour les politiques américaines et internationales en matière de santé sexuelle, d'éducation sexuelle et de droits sexuels dans diverses communautés » (traduction de la rédaction).

${ }^{47}$ La question de la médecine sexuelle a été traitée en détail dans les deux publications ci-dessous. Nous ne faisons ici que resituer sa place dans le développement plus général de la recherche sur la sexualité : M. A. Perelman, "The history of sexual medicine », in D. L. Tolman, L. M. Diamond, J. A. Bauermeister, W. H. George, J. G. Pfaus et L. M. Ward (éds), APA handbook of sexuality and psychology (vol. 2 : Contextual approaches,), Washington, DC, US American Psychological Association, p. 137-179. A. GiAmI, « La médecine sexuelle : genèse d'une spécialisation médicale ? », Histoire, médecine et santé, 12, 2017, p. 131-147.

${ }^{48}$ L. TIEFER, « Sexology and the Pharmaceutical Industry: The Threat of Co-optation », The Journal of Sex Research, 37/3, 2000, p. 273-283 ; G. Hart et K. Wellings, " Sexual behaviour and its medicalisation: in sickness and in health ", British Medical Journal, 324 (7342), 2002, p. 896-900 ; R. Moyninan et B. Mintzes, Sex, Lies and Pharmaceuticals. How do drug companies plan to profit from female sexual dysfunction, Vancouver, Greystone Books, 2010 ; et A. Giami, «L'invention des médicaments des troubles féminins du désir : controverses autour de la sexualité féminine », in D. GARDEY et M. VuILle (dir.), Les Sciences du désir. La sexualité féminine de la psychanalyse aux neurosciences, Lormont, Éditions Le bord de l'eau, 2018, p. 107-125. 
Sexual Medicine (ISSM) remonte à 1978. Au-delà des traitements des troubles sexuels, il s'agit d'introduire la question de la sexualité dans tous les domaines de la médecine et notamment celui des maladies chroniques. La publication en 2004 du premier numéro du Journal of Sexual Medicine ${ }^{49}$ s'inscrit résolument dans le développement de cette nouvelle communauté épistémique :

The second is the expansion of basic science and clinical research to formally include all aspects of sexual medicine. Sexual medicine may be considered as the medical discipline that embraces the study, diagnosis and treatment of sexual health concerns of men and women. The mission of The Journal of Sexual Medicine is to publish manuscripts of the highest quality multidisciplinary basic science and clinical research to delineate and understand the scientific basis of male and female sexual function and dysfunction, to provide education to healthcare professionals in sexual medicine, and to promote the exchange of scientific information generated from experimental and clinical research ${ }^{50}$.

Là encore, on assiste à la création d'une nouvelle communauté épistémique, dans le champ de la médecine, qui comprend des figures charismatiques (Irwin Goldstein, notamment), la mise en place d'une organisation très structurée et le développement de tout un ensemble de revues scientifiques désormais soutenues par les plus grandes maisons d'édition scientifique. On y relève aussi une ambition de haute qualité scientifique, de pluridisciplinarité et en associant la science fondamentale, la pratique clinique et la formation professionnelle des médecins. Les dimensions biomédicale et clinique et la pathologie, abandonnées par les critical sexuality studies, viennent occuper ici la place centrale dans les objectifs scientifiques de la médecine sexuelle. Les aspects sociologiques et culturels continuent à être traités, dans le champ de la médecine sexuelle, comme éléments de contexte.

\section{De la sexologie à la santé sexuelle}

En 2005, lors du congrès de Montréal, l'Association mondiale de sexologie décide d'abandonner le terme de sexologie au profit de celui de santé sexuelle. On retrouve ici aussi le processus de construction d'une communauté épistémique avec une organisation internationale très structurée en fédérations régionales et sociétés nationales, l'organisation de congrès mondiaux tous les deux ans et le soutien d'une

${ }^{49}$ I. Goldstein, « The Inaugural Issue of The Journal of Sexual Medicine (JSM) », The Journal of Sexual Medicine, 1/1, 2004, p. 1-2.

${ }^{50}$ Ibid., p. 1.

« La seconde est l'expansion de la science fondamentale et de la recherche clinique pour intégrer tous les aspects de la médecine sexuelle. La médecine sexuelle peut être considérée comme la discipline médicale qui englobe l'étude, le diagnostic et le traitement des problèmes de santé sexuelle des hommes et des femmes. La mission de The Journal of Sexual Medicine est de publier des manuscrits de science fondamentale et de recherche clinique multidisciplinaire de la plus haute qualité afin de délimiter et de comprendre les bases scientifiques du fonctionnement et du dysfonctionnement sexuels masculins et féminins, de fournir une formation aux professionnels de la santé en matière de médecine sexuelle et de promouvoir l'échange d'informations scientifiques issues de la recherche expérimentale et clinique » (traduction de l'auteur). 
revue : l'International Journal of Sexual Health dirigée par Eli Coleman, professeur de psychologie et de santé sexuelle à l'Université du Minnesota et ancien président de la WAS. La WAS ambitionne pour sa part de couvrir l'ensemble du champ de la sexologie/ santé sexuelle en mettant l'accent sur les droits sexuels et l'éducation à la sexualité. Si la WAS est dotée d'un comité scientifique international et pluridisciplinaire qui valide ses prises de position, sa mission réside principalement dans l'intervention publique et politique, le partenariat avec les grandes organisations internationales telles que l'OMS, l'Unesco et les Nations unies en vue de la mise en place de programmes d'éducation et de prévention et pour la promotion des droits sexuels.

\section{Épilogue}

Nous avons tenté de retracer l'histoire de la recherche scientifique sur la sexualité au cours du $\mathrm{XX}^{\mathrm{e}}$ siècle en essayant d'identifier et de situer la place du courant de la sex research qui s'est auto-identifié initialement comme la principale communauté épistémique de ce champ de recherche. À la suite d'une préhistoire marquée par les développements de la Sexualwissenschaft en Allemagne dès la fin du XIX ${ }^{\mathrm{e}}$ siècle et du Comité pour la recherche sur les problèmes de la sexualité (1922-1963), la sex research en tant que courant spécifique s'est développée principalement aux États-Unis à partir de la fin des années 1950. Les principaux fondateurs de la sex research et, notamment, Hugo Beigel, Hans Lehfeld et Richard Green ont eu l'ambition d'unifier un champ diversifié et dispersé dans différentes disciplines et dans différents pays en créant un espace commun et unifié pour les recherches fondamentales menées dans le champ biomédical, les sciences sociales et la recherche en éducation et en thérapie sexuelles. Ce projet s'est traduit par la formation de communautés épistémiques autonomes réunies dans des associations vivant des cotisations de leurs membres, se retrouvant régulièrement dans des congrès, publiant leurs articles dans quelques revues conçues et longtemps dirigées par leurs membres fondateurs. Pendant une vingtaine d'années, cette communauté s'est organisée et développée, avant que le champ de la recherche scientifique sur la sexualité n'éclate littéralement en une myriade de communautés et de modèles épistémiques ancrés dans des organisations, des revues et des sociétés savantes et que le dispositif la sex research en arrive à ne plus représenter qu'un courant de recherche parmi d'autres, historiquement daté et situé, et désormais battu en brèche sur les fronts des sciences sociales et de l'activisme d'une part et de la médecine sexuelle et de la santé sexuelle d'autre part.

On a ainsi assisté à partir de la fin des années 1980 au lancement et au développement des « grandes enquêtes sur les comportements sexuels » réalisées en population générale en réponse à l'arrivée de l'épidémie de VIH et qui ont constitué en soi un paradigme revendiquant une filiation avec l'œuvre de l'un des pionniers de la sex research, Alfred Kinsey. Le développement de ces recherches a ensuite ouvert la porte aux recherches menées dans le champ des sciences sociales, plus centrées sur les représentations, les significations et les relations de la vie sexuelle et engagées dans la lutte pour la promotion des droits humains et contre les discriminations. On est ainsi passé d'un modèle de la sex research à ambition scientiste à un autre modèle des critical sexuality studies mettant plus l'accent sur les dimensions subjectives, culturelles et politiques de la sexualité que sur les comportements, les relations et le 
rapport au risque. Le courant des critical sexuality studies retrouve ainsi une certaine continuité avec la Sexualwissenschaft dans sa posture militante et l'intégration de l'action politique - lutte contre les discriminations et en faveur de l'émancipation des communautés identitaires et sexuelles. Parallèlement au développement de ce courant « social-constructionniste », le courant de recherches en médecine sexuelle impliquant l'endocrinologie, la physiologie, la neurologie, la pharmacologie et l'urologie, qui constituait une part non négligeable de la sex research, a commencé à se développer et a constitué ses propres communautés (associations, congrès et revues) ainsi qu'une forme de pluridisciplinarité interne à la médecine. Enfin, la santé sexuelle développe des approches originales en éducation et en prévention tout en tentant d'y inclure la perspective des droits sexuels. Dans un éditorial d'Archives of Sexual Behavior, Kenneth Zucker recense désormais 89 revues scientifiques (toutes dotées d'un Impact Factor), soit 13 de plus qu'en 2002. Il s'enorgueillit de constater qu'Archives bénéficie toujours du facteur d'impact le plus élevé parmi toutes les revues répertoriées et rappelle que les principales revues qui se sont développées au cours des dernières années ont été fondées et sont souvent toujours dirigées par d'anciens membres de l'Academy. Une façon de rappeler que l'influence intellectuelle de l'Academy et du courant de la sex research est toujours vivace ${ }^{51}$.

Au-delà d'une histoire intellectuelle des sciences de la sexualité, dont la sex research aura été l'un des moments fondateurs, cette étude peut nous aider à comprendre la genèse et le développement des communautés épistémiques, leur implication dans la demande et la commande sociale de leur époque et leurs transformations, fondées autant sur les avancées épistémiques réalisées à ce moment que sur l'évolution de la demande sociale et la formulation de nouveaux problèmes. La transformation de la communauté épistémique de la sex research peut ainsi être envisagée comme le passage d'une « communauté » à un "réseau » dans lequel émergent progressivement de nouvelles communautés en réponse à l'arrivée et à l'évolution de nouvelles demandes sociales et politiques formulées à l'égard des sciences de la sexualité. Alors que la communauté épistémique initiale de la sex research se présentait comme fondée sur la volonté de développer les connaissances scientifiques dans les meilleures conditions, les communautés qui émergent, principalement à l'occasion de l'arrivée de l'épidémie de VIH-sida mais aussi des mouvements féministes et LGBT, vont bénéficier des acquis de la sex research afin de développer de nouveaux paradigmes de recherche.

Encadré 3 : Chronologie de la sex research

\begin{tabular}{|l|l|l|}
\hline Année & Titre & Pays \\
\hline 1906 & $\begin{array}{l}\text { Iwan Bloch : (1906) Transl. eng. (1909) The Sexual Life of } \\
\text { Our Time in Its Relations to Modern Civilization }\end{array}$ & Allemagne \\
\hline $1919-1933$ & Institut für Sexualwissenschaft (Berlin) Magnus Hirschfeld & Allemagne \\
\hline $1922-1965$ & Committee for Research in Problems of Sex & États-Unis \\
\hline
\end{tabular}

${ }^{51}$ K. ZuCKer, "The 2020s: The Next Decade for the Archives of Sexual Behavior », Archives of Sexual Behavior, 49, 2020, p. 1-12. 


\begin{tabular}{|c|c|c|}
\hline 1938 & $\begin{array}{l}\text { Premières ébauches de Kinsey - financées par le Committee } \\
\text { for Research in Problems of Sex }\end{array}$ & \\
\hline 1947 & Fondation du Kinsey Institute à Bloomington (Indiana) & États-Unis \\
\hline 1957 & $\begin{array}{l}\text { Society for the Scientific Study of Sexuality (4S) } \\
\text { Fondateurs de la } 4 \text { S : Hugo Beigel, Albert Ellis, Henry Guze, } \\
\text { Robert V. Sherwi, Hans Lehfeld }\end{array}$ & États-Unis \\
\hline 1963 & $\begin{array}{l}\text { Advances in Sex Research, vol. } 1 \text { (oct. 1963), p. XIII-XIV - } \\
\text { Hugo G. Belgel }\end{array}$ & États-Unis \\
\hline 1965 & $\begin{array}{l}\text { The Journal of Sex Research, vol. 1, no. } 1 \text { (mar. 1965) - Hans } \\
\text { Lehfeldt }\end{array}$ & États-Unis \\
\hline 1971 & $\begin{array}{l}\text { Archives of Sexual Behavior: An Interdisciplinary Research } \\
\text { Journal - Richard Green }\end{array}$ & États-Unis \\
\hline 1974 & Journal of Homosexuality - John de Cecco & États-Unis \\
\hline 1974 & $1^{\text {er }}$ Congrès international de sexologie médicale (Paris) & France \\
\hline 1975 & $\begin{array}{l}\text { First Annual meeting of the International Academy of Sex } \\
\text { Research (Stony Brooks, NY, USA) }\end{array}$ & États-Unis \\
\hline 1978 & $\begin{array}{l}\text { WAS : Premier congrès de la World Association for Sexology } \\
\text { (Rome) }\end{array}$ & Italie \\
\hline 1990 & $\begin{array}{l}\text { Annual Review of Sex Research (Society for the Scientific } \\
\text { Study of Sexuality) }\end{array}$ & États-Unis \\
\hline 1994 & $\begin{array}{l}\text { NHSLS : Laumann, E., Gagnon, J., Michael, R., \& Michaels, } \\
\text { S. (1994). The social organization of sexuality. Sexual } \\
\text { practices in the United States. Chicago: The University of } \\
\text { Chicago Press. }\end{array}$ & États-Unis \\
\hline 1995 & $\begin{array}{l}\text { Di Mauro, D. (1995). Sexuality Research in the United States. } \\
\text { An Assessment of the Social and Behavioral Sciences. New } \\
\text { York: SSRC. }\end{array}$ & États-Unis \\
\hline 1998 & Sexualities - Kenneth Plummer & $\begin{array}{l}\text { Grande- } \\
\text { Bretagne }\end{array}$ \\
\hline 1999 & Culture, Health and Sexuality - Peter Aggleton & $\begin{array}{l}\text { Grande- } \\
\text { Bretagne }\end{array}$ \\
\hline 2002 & $\begin{array}{l}\text { Archives of Sexual Behavior: arrivée de Kenneth Zucker } \\
\text { (éditeur en chef) }\end{array}$ & États-Unis \\
\hline 2004 & Journal of Sexual Medicine - Irwin Goldstein & États-Unis \\
\hline 2004 & Sexuality Research and Social Policy - Gilbert Herdt & \\
\hline 2005 & $\begin{array}{l}\text { La WAS adopte le nom de « World Association for Sexual } \\
\text { Health »-Congrès de Montréal }\end{array}$ & \\
\hline 2007 & $\begin{array}{l}\text { International Journal of Sexual Health - Eli Coleman } \\
\text { (remplace le Journal of Psychology \& Human Sexuality } \\
\text { fondé en 1988). }\end{array}$ & \\
\hline
\end{tabular}




\section{Bibliographie}

Aberle, S. D. et Corner, G. W., Twenty-Five Years of Sex Research: History of the National Research Council Committee for Research in Problems of Sex 19221947, Philadelphia, PA, W.B. Saunders Company, 1953.

Aggleton, P., Kippax, S., Mane, P., Parker, R. et de Zalduondo, B., « Editorial », Culture, Health and Sexuality, 1/1, 1999, p. 1-2.

Allyn, D., Make love not war. The Sexual Revolution: an unfettered history, Boston, Little, Brown \& Company, 2000.

Bancroft, J., "Alfred C. Kinsey and the Politics of Sex Research », Annual Review of Sex Research, 15, 2004, p.1-39.

Bayer, R., Homosexuality and American Psychiatry. The Politics of Diagnosis, New York, Basic Books, 1981.

Belgel, H. G., « Preface », Advances in Sex Research, 1, 1963, p. 14-15.

Benjamin, H., The Transsexual Phenomenon, New York, Julian Press, 1966.

Bullough, V. L., « Katharine Bement Davis: Sex research and the Rockefeller Foundation », Bulletin of the History of Medicine, 62/1, 1988, p. 74-89.

Bullough, V. L., "The Rockefellers and Sex Research », The Journal of Sex Research, 21/2, 1985, p. 113-125.

Bullough, V., «American Physicians and Sex Research and Expertise, 1900-1990», Journal of the History of Medicine and Allied Sciences, 52/2, 1997, p. 236-253.

Catania, J. Y., Moskowitz, J. T., Ruiz, M. et Cleland, J., « A review of national AIDS-related behavioral surveys », AIDS, 10-Suppl. A, 1996, p. 183-190.

Di Mauro, D., Sexuality Research in the United States. An Assessment of the Social and Behavioral Sciences, New York, Social Sciences Research Council, 1995.

Dose, R., « The World League for Sexual Reform: Some Possible Approaches », Journal of the History of Sexuality, 12/1, 2003, p. 1-15.

DowsETt, G., " The price of pulchritude, the cost of concupiscence: how to have sex in late modernity », Culture, Health and Sexuality, 17-Suppl. 1, 2015, p. 5-19.

Escoffier, J., Sexual Revolution, New York, Thunder's Mouth Press, 2003.

FERRARI, R., «Writing narrative style literature reviews », Medical Writing, 24/4, 2015 , p. 230-235.

GagnON, J., "Gender preferences in erotic relations: The Kinsey scale and sexual scripts », in McWhirter, D., SAnders, S. A. et ReInisch, J. M. (dir.), Homosexuality/Heterosexuality: Concepts of sexual orientation, New York, Oxford University Press, 1990, p. 177-207.

Gagnon, J., "Sex research and social change ", Archives of Sexual Behavior, 4/2, 1975, p. 111-141.

Giami, A., « De Kinsey au sida : l'évolution de la construction du comportement sexuel dans les enquêtes quantitatives », Sciences sociales et santé, IX/4, 1991, p. 23-56.

Giami, A., « Ethnographie d'une conférence médico-scientifique : l'influence de l'industrie pharmaceutique dans le champ de la sexologie », Revue Sociologie / Santé, 30, 2009, p. 187-210.

Giami, A., «L'invention des médicaments des troubles féminins du désir : controverses autour de la sexualité féminine », in GARDEY, D. et VuILle, M. (dir.), Les Sciences 
du désir. La sexualité féminine de la psychanalyse aux neurosciences, Lormont, Éditions Le bord de l'eau, 2018, p. 107-125.

Giami, A., « La médecine sexuēlle : genèse d'une spécialisation médicale ? », Histoire, médecine et santé, 12, 2017, p. 131-147.

Giami, A. et Schiltz, M.-A., " Representations of Sexuality and relations between partners: Sex research in France in the era of AIDS », Annual Review of Sex Research, 7, 1996, p. 125-157.

GoldsteIn, I., " The Inaugural Issue of The Journal of Sexual Medicine (JSM) », The Journal of Sexual Medicine, 1/1, 2004, p. 1-2.

Green, R., «A statement of purpose », Archives of Sexual Behavior, 1/1, 1971, p. I.

GreEn, R., "Hugh Hefner, the International Academy of Sex Research, and Its Founding President », Archives of Sexual Behavior, 46, 2017, p. 2211-2212.

GreEN, R., "The International Academy of Sex Research. In the Beginning ", Archives of Sexual Behavior, 14/4, 1985, p. 293-302.

Hart, G. et Wellings, K., « Sexual behaviour and its medicalisation: in sickness and in health », British Medical Journal, 324 (7342), 2002, p. 896-900.

Hegarty, P., « Beyond Kinsey: The Committee for Research in Problems of Sex and American Psychology », History of Psychology, 15/3, 2012, p. 197-200.

Heкma, G. et Giami, A. (dir.), Sexual Revolutions, Houndmills, Basingstoke, Palgrave, 2014.

Herdt, G., «A welcome from the editor », Sexuality Research and Social Policy, 1/1, 2004, p. 1-6.

Holzner, B. et Marx, J., Knowledge Application: The Knowledge System in Society, Boston, Allyn and Bacon, Inc., 1979.

Irvine, J., Disorders of Desire. Sexuality in Modern American Sexology, Philadelphia, Temple University Press, 2005.

JONES, A., " Sex is not a problem: The erasure of pleasure in sexual science research », Sexualities, 22/4, 2019, p. 643-668.

Laumann, E., Gagnon, J., Michael, R. et Michaels, S., « A political history of the National Sex Survey of Adults », Family Planning Perspectives, 261, 1994, p. 34-38.

Masters, W. et Johnson, V., Human sexual response, Boston, Little, Brown and $\mathrm{C}^{\mathrm{o}}$, 1966.

Meyer, M. et Molyneux-Hodgson, S., " Introduction: The Dynamics of Epistemic Communities ", Sociological Research Online, 15/2, 2011, http://www.socresonline. org.uk /15/2/14.html, 10.5153/sro.

Moynihan, R. et Mintzes, B., Sex, Lies and Pharmaceuticals. How do drug companies plan to profit from female sexual dysfunction, Vancouver, Greystone Books, 2010.

Oosterhuis, H., Stepchildren of nature. Krafft-Ebing, psychiatry and the making of sexual identity. Chicago, The University of Chicago Press, 2000.

Perelman, M. A., " The history of sexual medicine », in Tolman, D. L., Diamond, L. M., Bauermeister, J. A., George, W. H., Pfaus, J. G. et Ward, L. M. (éds), APA handbook of sexuality and psychology (vol. 2 : Contextual approaches,), Washington, DC, US American Psychological Association, p. 137-179. 
Plummer, K., « Introducing sexualities (editorial) », Sexualities, 1/1, 1998, p. 5-10.

ReIss, I., An Insider's View of Sexual Science since Kinsey, Lanham, Rowman \& Littlefield Inc., 2006.

Robinson, P., The modernization of sex, New York, Harper \& Row, 1976.

TAMAGNe, F., « La Ligue mondiale pour la réforme sexuelle : la science au service de l'émancipation sexuelle? », Clio, 22, 2005, p. 101-121.

Tiefer, L., « Sexology and the Pharmaceutical Industry: The Threat of Co-optation », The Journal of Sex Research, 37/3, 2000, p. 273-283.

ZuCKER, K., "From the Editor's Desk: Receiving the torch in the Era of Sexology Renaissance », Archives of Sexual Behavior, 31/1, 2002, p. 1-6.

Zucker, K., "The 2020s: The Next Decade for the Archives of Sexual Behavior ", Archives of Sexual Behavior, 49, 2020, p. 1-12. 